J. Clin. Chem. Clin. Biochem.

Vol. 26, 1988, pp. 105-115

(C) 1988 Walter de Gruyter \& Co. Berlin - New York

\title{
Untersuchungen zur Biotransformation von Meclozin im menschlichen Körper
}

Von S. Goenechea,

Institut für Rechtsmedizin der Universität Bonn, Bonn, Bundesrepublik Deutschland

G. Rücker,

Pharmazeutisches Institut der Universität Bonn, Bundesrepublik Deutschland

H. Brzezinka, G. Hoffmann,

Institut für Rechtsmedizin der Universität Bonn, Bonn, Bundesrepublik Deutschland

M. Neugebauer

Pharmazeutisches Institut der Universität Bonn, Bundesrepublik Deutschland und

G. Glanzmann ${ }^{1}$

Institut für Rechtsmedizin der Universität Bonn, Bonn, Bundesrepublik Deutschland

(Eingegangen am 22. Juni/21. Dezember 1987)

Zusammenfassung: Nach Einnahme von 1-[(4-Chlorphenyl)-phenylmethyl]-4-[(3-methylphenyl)-methyl]-piperazin (1, Meclozin) konnten im menschlichen Harn und Stuhl elf Verbindungen nachgewiesen werden, deren chemische Struktur mit Hilfe spektroskopischer Untersuchungen diskutiert und durch Vergleich mit den synthetisierten Substanzen bewiesen wurde:

Meclozin (1),

$\mathrm{N}-[(4-C h l o r p h e n y l)$-phenylmethyl]-piperazin (2),

3-\{4-[(4-Chlorphenyl)-phenylmethyl]-piperazino\}-methylbenzoesäure (3),

3-\{4-[(4-Chlorphenyl)-phenylmethyl]-piperazino\}-methylbenżoesäureamid (4),

2-〈3-\{4-[(4-Chlorphenyl)-phenylmethyl]-piperazino\}-methylbenzoyl $\rangle$-aminoethansulfonsäure (5),

3-\{4-[(4-Chlorphenyl)-3'-hydroxy-4'-methoxyphenylmethyl]-piperazino\}-methylbenzoesäure (6),

1-[(4-Chlorphenyl)-phenylmethyl] 4 -[(3-methylphenyl)-methyl]-piperazin- $\mathrm{N}^{4}$-oxid (7),

1-[(4-Chlorphenyl)-phenylmethyl]-4-[(3-methylphenyl)-methyl]-piperazin- $\mathrm{N}, \mathrm{N}^{\prime}$-dioxid (8),

3-Methylbenzoesäure (9),

3-Methylhippursäure $(10)$ und

3-Methylbenzoesäure-Glucuronid (11).

Der Nachweis von 11 erfolgte nach enzymatischer Spaltung durch Nachweis des Aglykons. Es ergaben sich Hinweise auf einen weiteren Metaboliten, dessen Identifizierung nicht gelang.

1 Aus der Dissertation, Universität Bonn, 1987. 


\section{Investigation of the biotransformation of Meclozine in the human body}

Summary: After oral administration of 1-[(4-chlorophenyl)-phenylmethyl]-4-[(3-methylphenyl)-methyl]-piperazine ( 1 , Meclozine) eleven compounds were isolated from human urine and faeces. The structural elucidation of the metabolites was accomplished by comparison of their spectral data with those of the synthetic reference compounds. The metabolites were identified as:

Meclozine (1),

$\mathrm{N}-[(4-$ chlorophenyl)-phenylmethyl]-piperazine (2),

3-\{4-[(4-chlorophenyl)-phenylmethyl]-piperazino\}-methyl-benzoic acid (3),

3-\{4-[(4-chlorophenyl)-phenylmethyl]-piperazino\}-methyl-benzamide (4),

2-〈3-\{4-[(4-chlorophenyl)-phenylmethyl]-piperazino\}-methyl-benzoyl $\rangle$-amino-ethanesulphonic acid (5),

3-\{4-[(4-chlorophenyl)-3'-hydroxy-4'-methoxyphenylmethyl]-piperazino\}-methyl-benzooic acid (๑),

1-[(4-chlorophenyl)-phenylmethyl]-4-[(3-methylphenyl)-methyl]-piperazine- $\mathrm{N}^{4} \div$ oxide (7),

1-[(4-chlorophenyl)-phenylmethyl]-4-[(3-methylphenyl)-methyl]-piperazine-N, $\mathrm{N}^{\prime}$-dioxide (8),

3-methyl-benzoic acid (9),

3-methyl-hippuric acid (10) and

3-methyl-benzoic acid-glucuronide (11).

The structure of compound 11 was confirmed after enżymatic cleavage and identification of the aglycon. A further metabolite was detected, but not identified.

\section{Einführung}

Racemisches Meclozin (1) findet in Form des Dihydrochlorides Anwendung als Antiallergikum, Antiemetikum und als Sedativum. Untersuchungen zum Metabolismus wurden bislang nur an Ratten durchgeführt $(1,2)$. Dabei konnten N-[(4-Chlorphenyl)phenylmethyl]-piperazin (2) und N-[(4-Chlorphenyl)phenylmethyl]-ethylendiamin als Abbauprodukte identifiziert werden.

Die vorliegende Arbeit berichtet über Nachweis, Isolierung und Identifizierung von Ausscheidungsprodukten im menschlichen Harn und Stuhl nach oraler Applikation von Meclozin-dihydrochlorid in Form von Postafen ${ }^{\oplus}$-Tabletten ${ }^{2}$.

\section{Methodik}

Eine gesunde, freiwillige, weibliche Versuchsperson (27 Jahre alt) nahm in mehreren Zeiträumen jeweils 10 Tage lang täglich 4 Postafen ${ }^{\circledast}$-Tabletten (100 mg Meclozin-dihydrochlorid) ein. Harn und Fäces wurden während der Versuchsdauer und darüberhinaus bis einschließlich 3 Tage nach Einnahmeende gesammelt. Während der Einnahme von Meclozin wurden andere Arzneimittel, Methylxanthin-haltige Lebensmittel, Nikotin und Alkohol ausgeschlossen. Der Harn wurde ohne Zusätze in braunen Glasflaschen bei $2-4^{\circ} \mathrm{C}$ im Kühlschrank aufbewahrt. Die Fäces wurden in Polyethylenflaschen gesammelt, mit Methanol versetzt und bis zur Aufbereitung ebenfalls im Kühlschrank aufbewahrt.

Vor Beginn der Medikamenteneinnahme wurden zu Vergleichszwecken 3 Liter Leerurin gesammelt, die wie die medikamentenhaltigen Proben aufbereitet wurden.

$\left.{ }^{2}\right)$ Hersteller: UCB Chemie GmbH, 5014 Kerpen.

\subsection{Aufbereitung des Harns}

Der nach einer Einnahmeperiode (10 Tage) erhaltene Sammelharn (etwa 14 Liter) wurde analog des in Abbildung 1 wiedergegebenen Extraktionsschemas aufbereitet. Die Extraktion erfolgte in einem 4 Liter Harn fassenden Perforator; die Extraktionszeit betrug jeweils 10 Stunden.

\subsection{Aufbereitung der Fäces}

Ausgangsmaterial für die Aufbereitung war die nach Zerkleinern der Fäces und Abzentrifugieren der festen Bestandteile erhaltene methanolische Stuhllösung. Diese wurde nach dem in Abbildung 2 dargestellten Extraktionsschema aufgearbeitet.

2.3. Gewinnung und enzymatische Spaltung der Glucuronide

Aus 14 Liter Harn, der zuvor zur Abtrennung der nicht-konjugierten Metaboliten nach Abbildung 1 extrahiert worden war, wurde durch Fällung mit Bleiacetat $(3,4)$ eine GlucuronidFraktion gewonnen. Diese wurde in $50 \mathrm{ml}$ Pufferlösung pH 5,5 (18 g/l Natriumacetatlösung, mit konz. Phosphorsäure auf $\mathrm{pH}$ 5,5 eingestellt) aufgenommen, mit $7 \mathrm{mg} \beta$-Glucuronidase (,from bovine liver"; SERVA, Feinbiochemika, Heidelberg) versetżt und eine Woche lang bei $37^{\circ} \mathrm{C}$ inkubiert. Anschließend wurde die wäßrige Phase nach Abbildung 1 extrahiert.

\subsection{Säulenchromatographie}

Reinigung und Auftrennung der Stuhlextrakte: Glassäule von $600 \mathrm{~mm}$ Länge und $25 \mathrm{~mm}$ Weite;

Sorbens: Kieselgel 60 , Korngröße $0.063-0.200 \mathrm{~mm}, 70-230$ mesh ASTM (E. Merck, Darmstadt);

Elutionsmittel: Dichlormethan bzw. Essigester, Steigerung der Polarität durch Dichlormethan (Essigester)/Methanol-Mischungẻn mit zunehmendem Methanolanteil bis zu reinem Methanol.

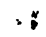


Harn

$+\mathrm{NaOH}(100 \mathrm{~g}$ ll) bis

pll 12

+ nellexan

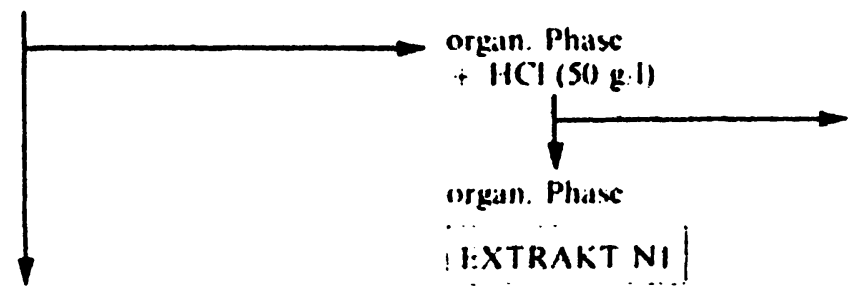

wäBrige Phase

$+\mathrm{NaOll}(100 \mathrm{~g} / \mathrm{l})$ bis

pH 12

+. Dichlormethan

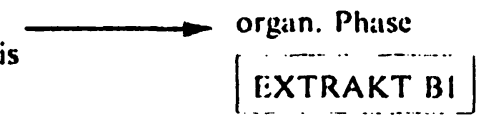

Metabolit ? und 4

wäBrige Phase

+ Dichlormethan

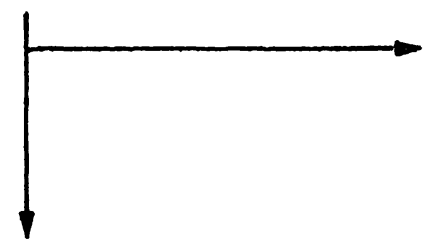

organ. Phase

$+H(1(5) g-1)$

wäbrige Phase

$+\mathrm{NaOH}(100 \mathrm{~g} / \mathrm{l})$ bis

organ. Phase

organ Phast pH 12

EXTRAKT N2

+ Dichlormethan

wäBrige Phasc

$+H(I(100$ g. I) bis

Metabolit 5

pH 8.5

+ Dichlormethan

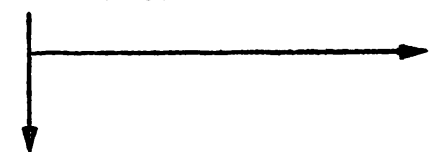

organ. Phase

EXTRAKT A

WäBrige Phase

$+\mathrm{HCl}(100 \mathrm{~g}: \mathrm{l})$ bis

Metabolit 3

$\mathrm{pH} I$

+ Dichlormethan

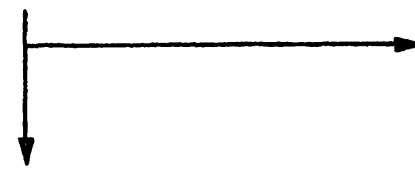

organ. Phase

\section{EXTRAKT S}

wäBrige Phase

Metabolit 9 und 10

Abb. 1. Extraktionsschema des Harns

\subsection{Dünnschichtchromatographie}

Es wurden Fertigplatten der Fa. Merck, Darmstadt verwendet: Kieselgel $60 \mathrm{~F}_{254}$, Schichtdicke $0.25 \mathrm{~mm}(200 \times 200 \mathrm{~mm}, 200$ $\times 100 \mathrm{~mm}, 50 \times 100 \mathrm{~mm})$, Schichtdicke $2 \mathrm{~mm}(200 \times 200$ $\mathrm{mm}$ ). Für die präparative Schichtchromatographie wurden die Platten vor Gebrauch in Methanol/Ammoniaklösung 25\% $(100+1$, Volumina) vorgereinigt und anschließend zwei Stunden im Trockenschrank bei $110^{\circ} \mathrm{C}$ aktiviert.

\section{Fließmittel}

1. Toluol/Ethanol/Eisessig (60 $+5+35$, Volumina)

2. Dichlormethan/Methanol/Ammoniaklösung $25 \%(90+10$ $+0,1$, Volumina)

3. n-Butanol/Ethanol/Wasser/Ammoniaklösung $25 \%$ (50 + 20 $+10+10$, Volumina)

4. 2-Propanol/Chloroform/Wasser/Ammoniaklōsung 25\% (64 $+32+2+2$, Volumina)

5. Methanol/Ammoniaklösung $25 \%(100+1$, Volumina)

6. Chloroform/Essigester/Methanol $(50+25+25$, Volumina).

\section{Detektionsmittel}

1. Kaliumiodoplatinat (5)

Nachbehandlung mit einer Mischung von $10 \mathrm{ml}$ wäßriger Eisen-(III)-chloridlösung (50 g/l) und $1 \mathrm{ml}$ konz. Schwefelsäure.

2. 2,6-Dichlorchinonchlorimid, $1 \mathrm{~g} / \mathrm{l}$ in Ethanol (5)

3. Altmann-Reagenz (6): 1 Teil einer Lösung von $50 \mathrm{~g} / 1$ 4Dimethylaminobenzaldehyd in Essigsäureanhydrid wird mit 4 Teilen Aceton gemischt. Nach Besprühen wird das Chromatogramm $10 \mathrm{~min}$ auf $100^{\circ} \mathrm{C}$ erhitzt.

4. Bromkresolgrün-Bromphenolblau-KaliumpermanganatReagenz (5)

Die Detektion der Metaboliten auf den präparativen Platten erfolgte unter UV-Licht oder durch Besprühen der Plattenränder mit dem geeigneten Reagenz. Nach Abtragen der substanzhaltigen Zonen wurde das Kieselgel auf eine in einer Glassäule befindlichen Schicht geglühten Seesandes gegeben (7). Die Elution der Substanzen erfolgte mit Dichlormethan/Methanol-Gemischen. Zum Abtrennen von Kieselgel wurde das Lösungsmittelgemisch im Stickstoffstrom zur Trockne eingedampft, der Rückstand in wenig Dichlormethan aufgenommen und durch ein Millipore-Filter (Millipore GmbH, Eschborn) filtriert. 


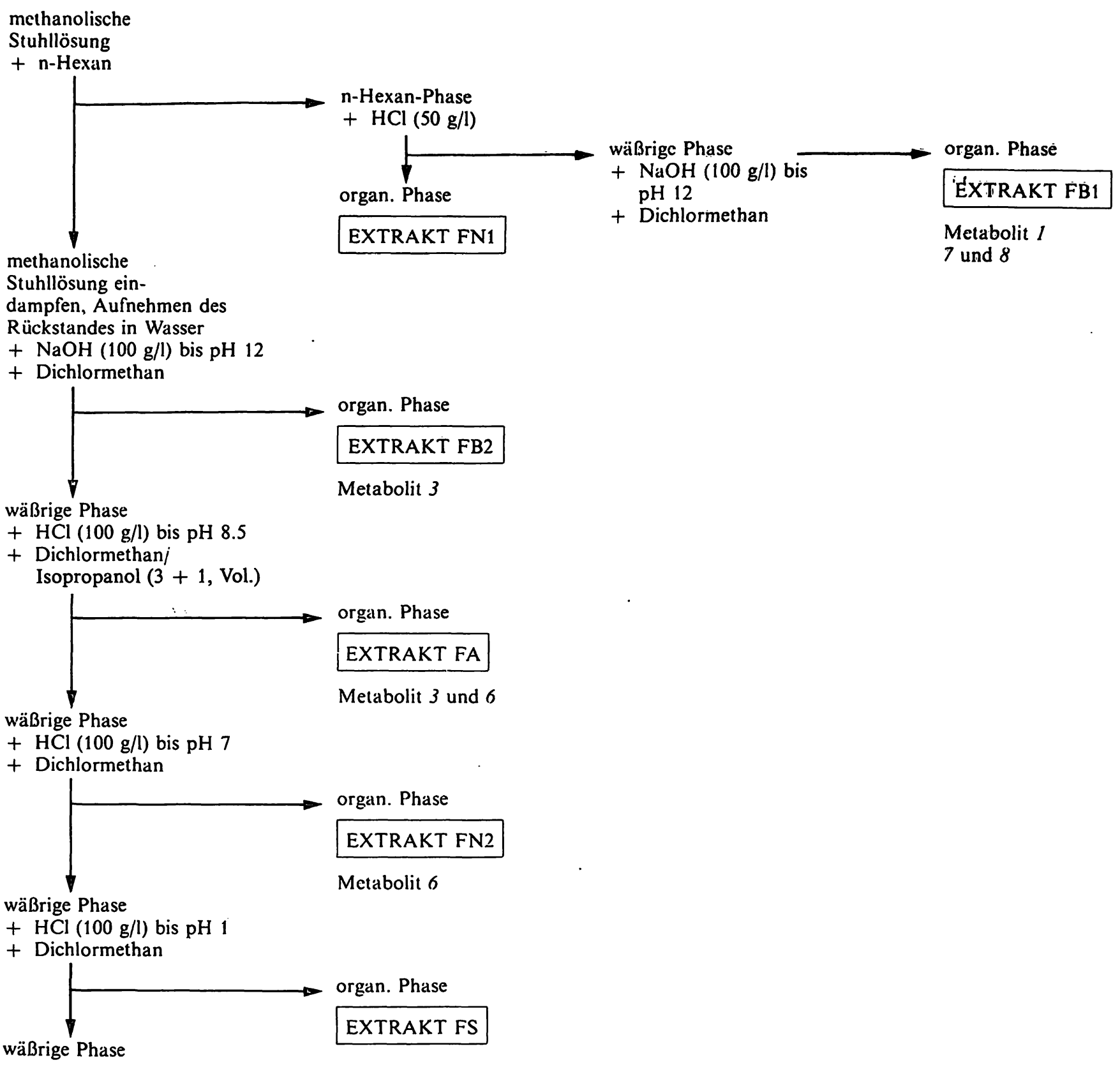

Abb. 2. Extraktionsschema der Fäces

2.6 Gaschromatographischer Nachweis von 3-Methylbenzoesäuremethylester neben Benzoesäuremethylester

Aus dem sauren Urinextrakt wurde mittels präparativer Schichtchromatographie ein Benzoesäure/3-Methylbenzoesäure-Gemisch isoliert. Nach Umsetzung mit etherischer Diazomethanlösung (8) wurden die Methylester gaschromatographisch vermessen:

Gepackte Säule, $2.0 \mathrm{~m}$ lang, $3.0 \mathrm{~mm}$ i.D.;

Füllung GP 2\% SP-2110, $1 \%$ SP-2510 DA auf Supelcoport 100/120 mesh;

Trägergas̀: $\mathrm{N}_{2}$;

Injektionsblock: $210^{\circ} \mathrm{C}$;

Detektor: $220^{\circ} \mathrm{C}$;

Ofen: $115^{\circ} \mathrm{C}$ (isotherm);

Benzoesäuremethylester (Retentionszeit $=1,76 \mathrm{~min}$ );

3-Methylbenzoesäuremethylester (Retentionszeit $=2,88 \mathrm{~min}$ ).

\subsection{Geräte}

Massenspektren

Finnigan/MAT 212 und Datensystem SS 188 (Finnigan MAT $\mathrm{GmbH}$, Bremen); Ionisierungsenergie: $80 \mathrm{eV}$; Ionenquellentemperatur: $250^{\circ} \mathrm{C}$.

\section{FAB-Spektren}

MAT 731 und Datensystem SS 200; Beschleunigungṣspannung $8 \mathrm{kV}$; FAB-Kanone: ION-TEC Ltd, Modell $11 \mathrm{NF}$; Xe.

\section{GC/MS}

1. Finnigan/MAT 1020B; Kapillarsäule DB 5 (J \& W Scientific Inc., Rancho Cordova, USA), Fused silica, $30 \mathrm{~m} \times 0.32 \mathrm{~mm}$ 
i. D., Filmdicke: $0.25 \mu \mathrm{m}$, Trägergas: He, Split $1: 20$, dirckte Kopplung; MS: Ionenquellentemperatur: $180^{\circ} \mathrm{C}$, Ionisierungsenergie: $70 \mathrm{eV}$.

2. Finnigan/MAT 212; Kapillarsäule OV 1701 (Machercy-Nagel, Düren), Fused silica, $25 \mathrm{~m} \times 0.25 \mathrm{~mm}$ i. D., Filmdicke: $0.27 \mu \mathrm{m}$, Trägergas: $\mathrm{He}$, offene Kopplung; MS: Ionenquellentemperatur: $250^{\circ} \mathrm{C}$, Ionisierungsenergie: $80 \mathrm{cV}$.

\section{'H-NMR-Spektren}

WH 90 (Bruker, Analytische Meßtechnik GmbH, Rheinstetten), $90 \mathrm{MHz}$.

\section{IR-Spektren}

Spektrophotometer Modell 298 (Bodenscewerk Perkin Elmer \& Co. GmbH, Überlingen).

\section{UV-Spektren}

550 SE UV/VIS Spektrophotometer (Bodenseewerk Perkin Elmer \& Co. GmbH, Überlingen).

\subsection{Vergleichssubstanzen}

Meclozin (I): UCB Chemie GmbH, Kerpen; $\mathrm{N}$-[(4-Chlorphenyl)-phenylmethyl]-piperazin (2): Aldrich Chemie GmbH \& Co. KG, Steinheim;

3-Methylbenzoesäure (9): Merck-Schuchardt, Hohenbrunn; 3-Methylhippursäure (10) wurde analog l.c. (9) durch Umsetzung von 3-Methylbenzoylchlorid mit Glycin in natronalkalischem Milieu hergestellt;

die Methylester von 3-Methylbenzoesäure (9) und 3-Methylhippursäure (10) wurden durch Umsetzung von 9 bzw. $10 \mathrm{mit}$ etherischer Diazomethanlösung (8) hergestellt;

$\mathrm{N}$-[(4-Chlorphenyl)-phenylmethyl]-ethylendiamin wurde nach l.c. (2) durch Umsetzung von 4-Chlorbenzhydrylchlorid mit einem Überschuß an Ethylendiamin dargestellt.
Folgende Vergleichssubstanzen wurden erstmalig synthetisiert $(10,11)$ :

3-\{4-[(4-Chlorphenyl)-phenylmethyl]-piperazino\}-methylbenzoesäure (3),

3-\{4-[(4-Chlorphenyl)-phenylmetbyl]-piperazino\}-methylbenzoesäureamid (4),

2-〈3-\{4-[(4-Chlorphenyl)-phenylmethyl]-piperazino\}-methylbenzoyl $\rangle$-aminoethansulfonsäure (5),

die isomeren Verbindungen 3-\{4-[(4-Chlorphenyl)-3'-hydroxy4'-methoxyphenylmethyl]-piperazino\}-methylbenzocsäurc (G) und $3-\{4-[(4-C h l o r p h e n y l)-4 '-h y d r o x y-3 '$-methoxyphenylmethyl]-piperazino\}-methylbenzoesäure,

1-[(4-Chlorphenyl)-phenylmethyl]-4-[(3-methylphenyl)-methyl]piperazin- $\mathrm{N}^{4}$-oxid (7) und

1-[(4-Chlorphenyl)-phenylmethyl]-4-[(3-methylphenyl)-methyl]piperazin- $\mathrm{N}, \mathrm{N}^{\prime}$-dioxid (8).

\section{Ergebnisse}

Nach Einnahme von Meclozin-dihydrochlorid (1diHCl) konnten in Harn und Fäces zehn körperfremde Substanzen mit Hilfe der Dünnschichtchromatographie detektiert werden (Tab. 1). Fünf Metabolite $(2,4,5,9,10)$ konnten nur in den Harnextrakten, vier Metabolite $(1,6,7,8)$ nur in den Stuhlextrakten detektiert werden. Metabolit 3 trat sowohl in den Fäces als auch im Harn auf (Tab. 1). In den nach Spaltung der Glucuronide erhaltenen Harnextrakten konnten keine stickstoffhaltigen körperfremden Substanzen auf der Dünnschichtplatte erkannt werden. Lediglich im sauren Urinextrakt wurde nach enzymatischer Spaltung Metabolit 9 nachgewiesen. Die Identifizierung der durch präparative Schichtchromatographie bzw. im Falle der Stuhlextrakte durch Säu-

Tab. 1. Dünnschichtchromatographie von Meclozin (1) und seinen Metaboliten.

\begin{tabular}{|c|c|c|c|c|c|c|c|c|c|c|c|c|}
\hline \multirow{2}{*}{$\begin{array}{l}\text { Meta- } \\
\text { bolit }\end{array}$} & \multicolumn{2}{|c|}{ Vorkommen* } & \multicolumn{6}{|c|}{$\underline{R_{r} \text { Werte in Fließmittel }}$} & \multicolumn{4}{|c|}{ Anfärbung mit Sprühreagenz } \\
\hline & Harn & Fäces & 1 & 2 & 3 & 4 & 5 & 6 & 1 & 2 & 3 & 4 \\
\hline 1 & - & +++ & 0,6 & 1,0 & 1,0 & 1,0 & 1,0 & 1,0 & $\begin{array}{l}\text { Blau- } \\
\text { Violett }\end{array}$ & - & - & $\begin{array}{l}\text { Blau- } \\
\text { Violett }\end{array}$ \\
\hline 2 & +++ & - & 0,5 & 0,4 & 0,8 & 0,6 & 0,4 & 0,2 & $\begin{array}{l}\text { Blau- } \\
\text { Violett }\end{array}$ & Violett & - & $\begin{array}{l}\text { Blau- } \\
\text { Violett }\end{array}$ \\
\hline 3 & + & +++ & 0,6 & 0,1 & 0,6 & 0,3 & 1,0 & 0,4 & Blau & - & - & Blau \\
\hline 4 & ++ & - & 0,4 & 0,6 & 0,9 & 1,0 & 0,9 & 0,7 & Blau & - & - & Blau \\
\hline 5 & ++ & - & 0,1 & 0,1 & 0,6 & 0,4 & 1,0 & 0,2 & Blau & - & - & Blau \\
\hline 6 & - & + & 0,4 & 0,0 & 0,6 & 0,2 & 1,0 & 0,3 & Blau & Blau & - & Blau \\
\hline 7 & - & ++ & 0,7 & 0,5 & 1,0 & 1,0 & 0,8 & 0,6 & $\begin{array}{l}\text { Blau- } \\
\text { Violett }\end{array}$ & Hellgelb & Gelb & Violett \\
\hline 8 & - & + & 0,7 & 0,3 & 1,0 & 0,6 & 0,6 & 0,2 & $\begin{array}{l}\text { Blau- } \\
\text { Violett }\end{array}$ & - & Hellbraun & Violett \\
\hline 9 & + & - & 0,8 & 0,1 & 0,6 & 0,3 & 0,9 & 0,7 & & - & - & Gelb \\
\hline 10 & +++ & - & 0,5 & 0,0 & 0,5 & $.0,3$ & 1,0 & 0,1 & - & - & Orange & Hellgelb \\
\hline
\end{tabular}

$*++++$ sehr intensiv, +++ intensiv, ++ wenig intensiv, + Spuren 
lenchromatographie isolierten Verbindungen erfolgte mit Hilfe spektroskopischer Methoden und durch Vergleich mit den synthetisierten Substanzen.

Metabolit 1 (Abb. 9) wurde aufgrund des Fließverhaltens in unterschiedlichen Fließmitteln sowie aufgrund der spektroskopischen Daten als unveränderte Meclozin-Base identifiziert. Das Massenspektrum (Abb. 3) $(12,13)$ sowie das IR- und UV-Spektrum $(13,14)$ sind bereits bekannt. Das ${ }^{1} \mathrm{H}-\mathrm{NMR}$-Spektrum $\left(\mathrm{CDCl}_{3}\right)$ von 1 zeigt zwischen $\delta=7.37-7.07 \mathrm{ppm}$ ein Multiplett für 13 aromatische Protonen. Bei $\delta=4.18 \mathrm{ppm}$ erscheint das Methin-Proton als Singulett; die beiden Protonen der Methylenbrücke zeigen ein Signal bei $\delta=3.44 \mathrm{ppm}$. Die Protonen des Piperazin-Ringes erscheinen als verbreitertes, nicht aufgelöstes Signal bei $\delta=2.40 \mathrm{ppm}$; die Methylgruppe zeigt ein Singulett bei $\delta=2.29 \mathrm{ppm}$.

Metabolit 2 (Abb. 9) wurde anhand der spektroskopischen Daten (MS [15], IR [16], ${ }^{1} \mathrm{H}-\mathrm{NMR}$ [17], UV [1]) als $\mathrm{N}$-[(4-Chlorphenyl)-phenylmethyl]-piperazin identifiziert.

Das EI-Massenspektrum des Metaboliten 3 (Abb. 4) zeigt im Vergleich zu 1 ein um 30 Einheiten höheres Molekülion bei $\mathrm{m} / \mathrm{z}$ 420/422 (3\%/1\%). Dies ließ eine Oxidation der am Aromaten gebundenen Methylgruppe zur Carboxylgruppe vermuten. Demnach sollte es sich bei 3 um 3-\{4-[(4-Chlorphenyl)-phenylmethyl]-piperazino\}-methylbenzoesäure (Abb. 9) handeln. Im ${ }^{1} \mathrm{H}-\mathrm{NMR}$-Spektrum des Metaboliten erscheint das Multiplett der beiden zur Carboxylgruppe ortho-ständigen aromatischen Protonen bei $\delta=8.03-7.92 \mathrm{ppm}$. Da die Verbindung neben einer sauren Carboxylgruppe auch zwei basische Stickstoffatome enthält, ist sie in der Lage, innere Salze zu bilden. Die im Vergleich zum Meclozin-Spektrum beobachtete Tieffeldverschiebung der beiden Protonen der Methylenbrücke $(\delta=3.73 \mathrm{ppm})$ spricht für eine Protonierung des basischeren, benzylsubstituierten Stickstoffs (18). Das bei $\delta=8.35 \mathrm{ppm}$ auftretende Singulett wird dem Ammonium-Proton zugeordnet. Im IR-Spektrum von 3 ist die Carbonylabsorption bei $1710 \mathrm{~cm}^{-1}$ nur schwach ausgeprägt; dagegen können bei $1570 \mathrm{~cm}^{-1}$ und $1390 \mathrm{~cm}^{-1}$ die asymmetrische und symmetrische Valenzschwingung der ionisierten Carboxylatgruppe registriert werden. Das UV-Spektrum $(1 \mathrm{~mol} / 1 \mathrm{HCl})$ von 3 weist Absorptionsmaxima bei $\lambda=232,257,264$ und $270 \mathrm{~nm}$ auf.

Das EI-Massenspektrum des Metaboliten 4 (Abb. 5) zeigt große Ähnlichkeit mit dem Spektrum von 3. Sowohl das Molekülion m/z 419/421 als auch die durch Benzylspaltungen entstandenen Fragmente $\mathrm{m} / \mathrm{z}$ 134 und $\mathrm{m} / \mathrm{z} 218$ liegen um eine Masseneinheit niedriger als die entsprechenden Bruchstücke im Spek-

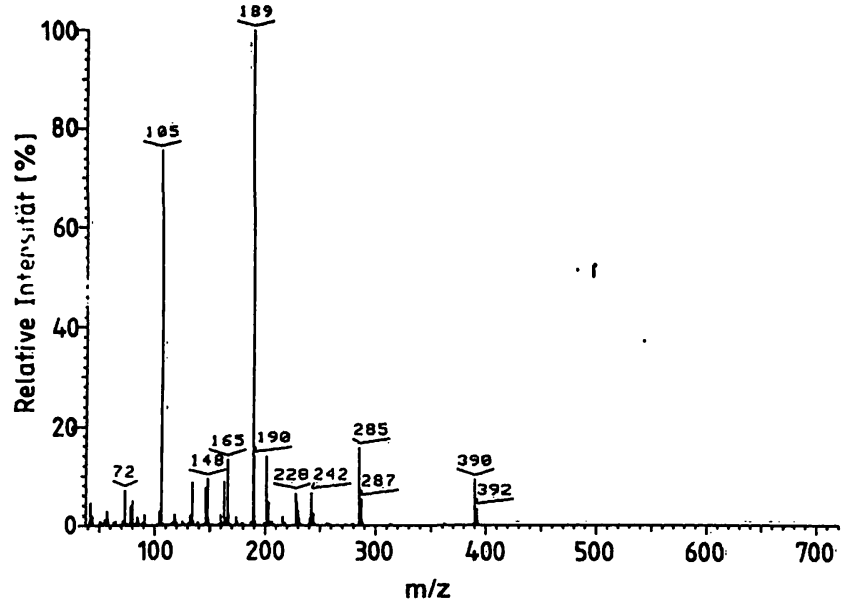

Abb. 3. EI-Massenspektrum von Meclozin (I)

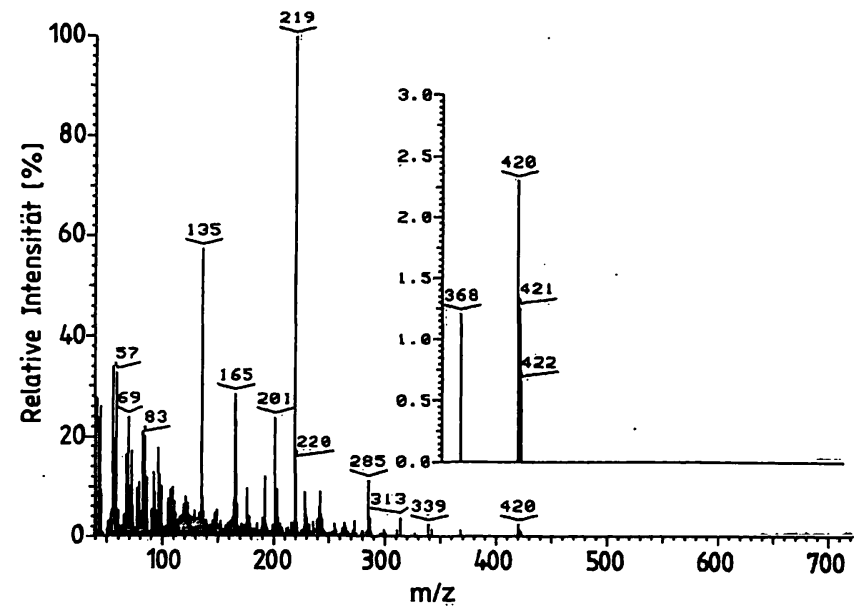

Abb. 4. EI-Massenspektrum von Metabolit 3

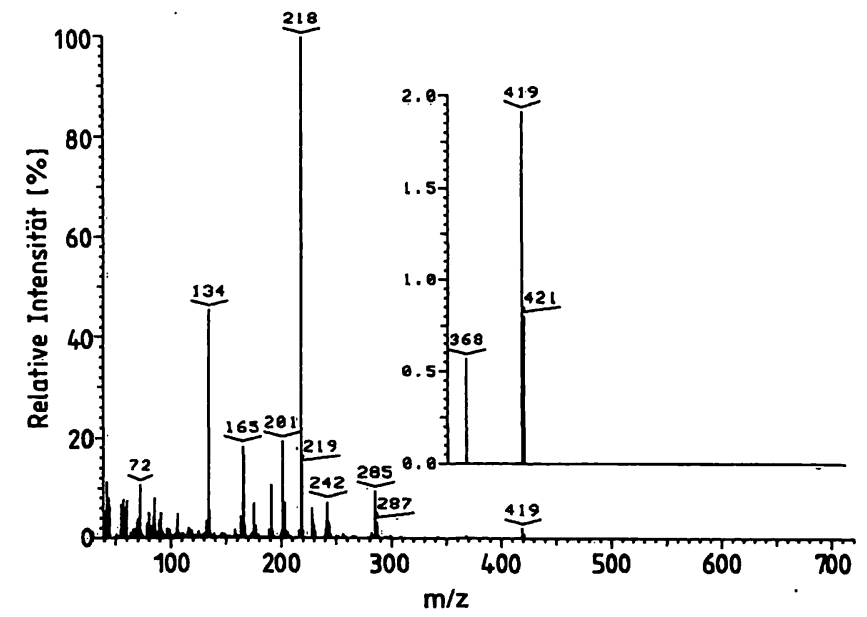

Abb. 5. EI-Massenspektrum von Metabolit 4

trum von 3. Nach Hochauflösung des Molekülpeaks $\left(\mathrm{m} / \mathrm{z} 419 / 421, \mathrm{C}_{25} \mathrm{H}_{26} \mathrm{ClN}_{3} \mathrm{O}\right)$ wurde das Vorliegen von 3-\{4-[(4-Chlorphenyl)-phenylmethyl]-piperazino\}-methylbenzoesäureamid (Abb. 9) vermutet. Im ${ }^{1} \mathrm{H}$ NMR-Spektrum $\left(\mathrm{CDCl}_{3}\right)$ der Verbindung wird das Multiplett der beiden zur Säureamidgruppe ortho= 
ständigen aromatischen Protonen bei $\delta=7.71-7.62$ ppm registriert. Das schwache, stark verbreiterte, mit $\mathrm{D}_{2} \mathrm{O}$ austauschbare Signal bei $\delta=5.78 \mathrm{ppm}$ wird den Amid-Protonen zugeordnet. Das IR-Spektrum von 4 zeigt NH-Valenzschwingungen bei $3360 \mathrm{~cm}^{-1}$ und $3200 \mathrm{~cm}^{-1}$. Bei $1670 \mathrm{~cm}^{-1}$ und $1610 \mathrm{~cm}^{-1}$ werden die $\mathrm{C}=\mathrm{O}$-Valenzschwingung bzw. die NH-Deformationsschwingung des Säureamids beobachtet. Das in Dichlormethan aufgenommene UV-Spektrum von 4 zeigt ein Absorptionsmaximum bei $\lambda=228 \mathrm{~nm}$.

Metabolit 5 wurde nach Spektrenvergleich mit der synthetisierten Vergleichssubstanz als Taurin-Konjugat von 3-\{4-[(4-Chlorphenyl)-phenylmethyl]-piperazino\}-methylbenzoesäure (3) identifiziert. Da sich die Verbindung im Massenspektrometer nicht unzersetzt verdampfen ließ, wurden zur Ermittlung des Molekülpeaks FAB-Spektren aufgenommen. Das PIFAB (Positiv Ion Fast Atom Bombardment)-Spektrum (Abb. 6) liefert in den Matrizes Thioglycerin und Glycerin ein Quasimolekülion von 5 bei $\mathrm{m} / \mathrm{z} 528 / 530$ $(100 \% / 42 \%)=[\mathrm{M}+\mathrm{H}]^{+}$. Bei m/z 550/552 (80\%/ $36 \%)$ und $\mathrm{m} / \mathrm{z} 572 / 574(22 \% / 9 \%)$ sind Clusterionen mit Natrium erkennbar. Das Auftreten zweier Clusterionen spricht für das Vorliegen von freier Sulfonsäure neben dem entsprechenden Natriumsalz. Das NIFAB (Negativ Ion Fast $A$ tom Bombardment)Spektrum liefert in der Matrix Glycerin das Quasimolekülion von 5 bei $\mathrm{m} / \mathrm{z} 526 / 528$ (100\%/42\%) $=[\mathrm{M}-\mathrm{H}]^{-}$. Das ${ }^{1} \mathrm{H}-\mathrm{NMR}-$ Spektrum $\left(\mathrm{d}_{4}-\mathrm{Methanol}\right)$ des Taurin-Konjugats zeigt große Ähnlichkeit mit dem Spektrum des Säureamids 4. Zusätzlich erscheinen die Methylenprotonen der Aminosäure als zwei zu Tripletts aufgespaltene Signale $(\mathrm{J}=7 \mathrm{~Hz})$ bei $\delta=3.75$ ppm $\left(-\mathrm{CH}_{2}-\mathrm{SO}_{3}-\right)$ und $\delta=3.03$ ppm $(-\mathrm{N}-$ $\mathrm{CH}_{2}$-). Das IR-Spektrum zeigt eine breite Absorptionsbande bei $3430 \mathrm{~cm}^{-1}(\mathrm{O}-\mathrm{H}, \mathrm{N}-\mathrm{H})$. Die $\mathrm{C}=\mathrm{O}-$ Valenz- und NH-Deformationsschwingung des Säureamids werden als Absorptionsbanden bei $1645 \mathrm{~cm}^{-1}$ und $1550 \mathrm{~cm}^{-1}$ erkannt. Die bei $1210 \mathrm{~cm}^{-1}$ und 1055 $\mathrm{cm}^{-1}$ auftretenden Absorptionsbanden werden den Schwingungen der Sulfonsäuregruppe zugeordnet. Das UV-Spektrum (Methanol) von 5 zeigt ein $\mathrm{Ab}$ sorptionsmaximum bei $\lambda=228 \mathrm{~nm}$.

Aufgrund eines Vergleichs mit den synthetisierten Isomeren wurde Metabolit 6 als 3-\{4-[(4-Chlorphenyl)3'-hydroxy-4'-methoxyphenylmethyl]-piperazino\}-methylbenzoesäure identifiziert (Abb. 9). Da 6 in paraStellung zur phenolischen OH-Gruppe nicht substituiert ist, kann die Verbindung mit 2,6-Dichlorchinonchlorimid (Detektionsmittel 2) auf der Dünnschichtplatte $\mathrm{zu}$ einem blauen Indophenolfarbstoff reagieren; die isomere Verbindung, das 4-Hydroxy-3methoxy-Derivat, kann in dieser Weise nicht mit dem
Reagenz reagieren. Im Massenspektrometer lassen sich die beiden isomeren Säuren nicht unzersetzt verdampfen. Sie wurden daher durch Umsetzung mit Diazomethan in die entsprechenden Methylester überführt. Der Methylester des Metaboliten 6 zeigt das Molekülion bei $\mathrm{m} / \mathrm{z}$ 480/482 (Abb. 7). Durch $\alpha$-Spaltung und Abspaltung eines $\cdot \mathrm{OCH}_{3}$-Radikals gelangt man zum Bruchstück m/z 449/451. Der weitere Abbau ist gekennzeichnet durch Benzylspaltungen. Im ${ }^{1} \mathrm{H}$ NMR-Spektrum ( $\mathrm{d}_{4}$-Methanol) von 6 liegen die Protonen des 1,3,4-trisubstituierten Benzolringes im Bereich $\delta=6.85-6.80 \mathrm{ppm}$ und zeigen das Bild zweier Singuletts. Im Spektrum der isomeren Verbindung liegen die entsprechenden Protonen im Bereich $\delta=6.93-6.62 \mathrm{ppm}$ und weisen eine andere Feinstruktur auf. Die zur Carboxylgruppe ortho-ständigen aromatischen Protonen erscheinen übereinstimmend als Multiplett bei $\delta=7.91-7.82 \mathrm{ppm}$; die übrigen aromatischen Protonen ergeben ein Multiplett zwischen $\delta=7.45$ und $\delta=7.18 \mathrm{ppm}$. Die Protonen der Methoxygruppe werden im Spektrum von 6 bei

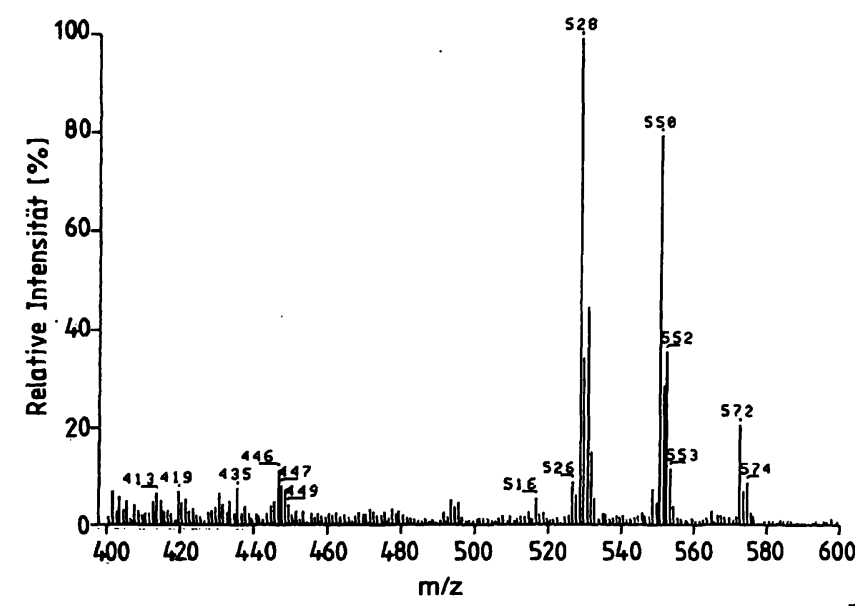

Abb. 6. Ausschnitt aus dem PIFAB-Spektrum (Thioglycerin) von Metabolit 5

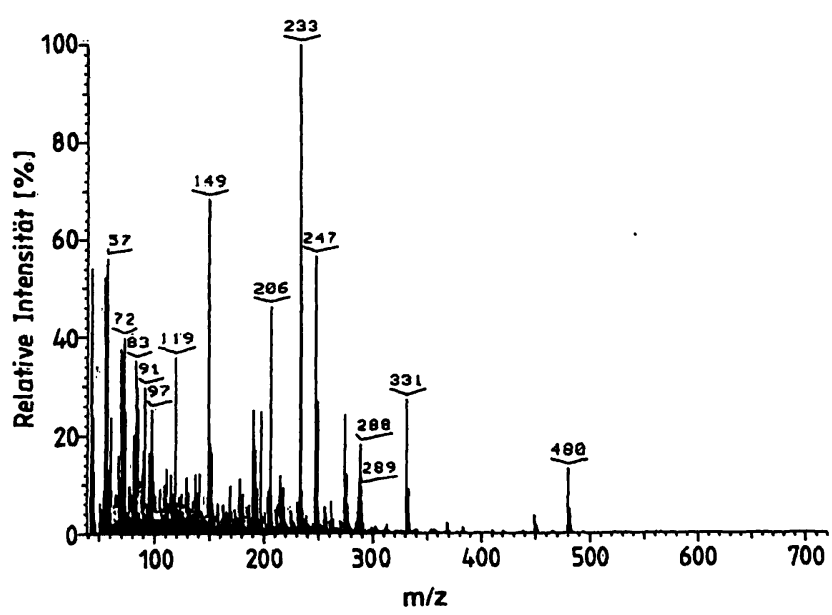

Abb. 7. EI-Massenspektrum von Metabolit 6 nach Derivatisierung zum Methylester 
$\delta=3.76 \mathrm{ppm}$, im Falle der 4-Hydroxy-3-methoxyVerbindung bei $\delta=3.78 \mathrm{ppm}$ registriert. Im IR-Spektrum von Metabolit 6 erscheint bei $3430 \mathrm{~cm}^{-1}$ die $\mathrm{O}-\mathrm{H}-$ Valenzschwingung. Das Fehlen einer Carbonylabsorption bei ca. $1700 \mathrm{~cm}^{-1}$ und das Auftreten zweier intensiver Banden bei $1570 \mathrm{~cm}^{-1}$ und 1390 $\mathrm{cm}^{-1}$ deuten, ähnlich wie bei Metabolit 3, auf das Vorliegen einer ionisierten Carboxylatgruppe in $6 \mathrm{hin}$. Das in Methanol aufgenommene UV-Spektrum von 6 zeigt ein Absorptionsmaximum bei $\lambda=282 \mathrm{~nm}$.

Das EI-Massenspektrum des als Meclozin- $\mathrm{N}^{4}$-oxid identifizierten Metaboliten 7 (Abb. 9) zeigt ein wenig intensives Molekülion bei $\mathrm{m} / \mathrm{z} 406 / 408$ (2.6\%/0.9\%). Das bei m/z 390/392 (2\%/0.75\%) auftretende Fragmention entsteht gemäß der Differenz von 16 Masseneinheiten durch Sauerstoff-Abspaltung aus dem Molekülion. Das ${ }^{1} \mathrm{H}-\mathrm{NMR}$-Spektrum $\left(\mathrm{CDCl}_{3}\right)$ von 7 zeigt große Ähnlichkeit mit dem des Meclozins (1). Das bei $\delta=4.34 \mathrm{ppm}$ registrierte Signal entspricht dem Methin-Proton und den beiden Protonen der Methylenbrücke. Im Vergleich zum ${ }^{1} \mathrm{H}-\mathrm{NMR}$-Spektrum des Meclozins (l) zeigen diese Protonen eine Verschiebung zum tieferen Feld. Da der Betrag der Tieffeldverschiebung für die Protonen der Methylenbrücke gegenüber der des Methinprotons wesentlich größer ist, wird das Vorliegen eines N-Oxides am zur Methylenbrücke benachbarten Stickstoff des Piperazin-Ringes angenommen. Im IR-Spektrum von 7 wird bei $955 \mathrm{~cm}^{-1}$ die $\mathrm{N}-\mathrm{O}-$ Valenzschwingung des $\mathrm{N}$-Oxides angeregt. Das UV-Spektrum $(1 \mathrm{~mol} / 1 \mathrm{HCl})$ zeigt Absorptionsmaxima bei $\lambda=232,260,264$ und $274 \mathrm{~nm}$.

Aufgrund der geringen isolierten Menge an Metabolit 8 war eine Identifizierung nur aufgrund des Massenspektrums möglich. Im EI-Massenspektrum ist ein schwaches Molekülion bei m/z 422 erkennbar. Abspaltung von Sauerstoff führt zu dem Fragment $\mathrm{m} / \mathrm{z}$ 406. Basispeak ist das durch Benzylspaltung entstandene Cl-haltige Bruchstück m/z 201/203. Die im Vergleich zu Meclozin (I) um 32 Einheiten höhere relative Molekülmasse des Molekülpeaks und die beobachteten Fragmentierungen machen für 7 das Vorliegen von 1-[(4-Chlorphenyl)-phenylmethyl]-4-[(3-methylphenyl)-methyl]-piperazin-N,N'-dioxid (Abb.

9) wahrscheinlich. Durch Aufnahme eines $\mathrm{CI}-\mathrm{NH}_{3}$-Massenspektrums (Abb. 8) konnte die Intensität des Quasi-Molekülpeaks (MH) ${ }^{+}$bei $\mathrm{m} / \mathrm{z}$ 423/425 wesentlich erhöht werden. Die Fragmentierung von 8 unter den Bedingungen der chemischen Ionisation entspricht weitgehend der durch Elektronenstoß induzierten Fragmentierung. Die räumliche Lage der Substituenten am Piperazin-Ring konnte nicht geklärt werden.

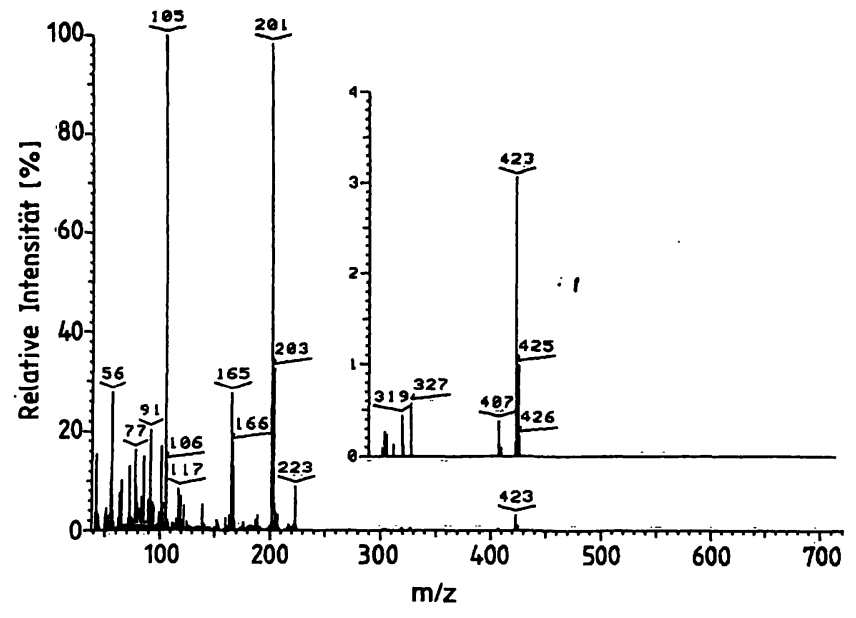

Abb. 8. CI-NH $\mathrm{NH}_{3}$-Massenspektrum von Metabolit 8

Zur Identifizierung des Metaboliten 9 (3-Methylbenzoesäure) wurde die gaschromatographische Retentionszeit und die GC/MS-Technik herangezogen. Eine dünnschichtchromatographische Unterscheidung zwischen 3-Methylbenzoesäure (9) (Abb. 9) und im Harn natürlich vorkommender Benzoesäure war aufgrund des $R_{1}$-Wertes nicht möglich. Daher wurde das aus dem sauren Harnextrakt durch präparative Schichtchromatographie isolierte Benzoesäure/3-Methylbenzoesäure-Gemisch nach Umsetzung zum Methylester gaschromatographisch getrennt. 3-Methylbenzoesäuremethylester wurde anschließend durch Massenchromatographie der Ionen $\mathrm{m} / \mathrm{z}$ 150, m/z 119 und $\mathrm{m} / \mathrm{z} 91$ detektiert und durch den Vergleich des Massenspektrums mit dem Spektrum der Referenzsubstanz identifiziert.

Metabolit 10 (Abb. 9) konnte als Glycin-Konjugat der 3-Methylbenzoesäure (9) identifiziert werden. Durch präparative Schichtchromatographie (Fließmittel 1) wurde 10 von der im Harn in großer Menge vorkommenden Hippursäure abgetrennt. Die Identifizierung von 10 erfolgte nach Derivatisierung zu 3Methylhippursäuremethylester. Das Massenspektrum des Methylesters ist bereits bekannt (19). Das ${ }^{1} \mathrm{H}$ NMR-Spektrum zeigt im Bereich $\delta=7.62-7.22$ ppm das Multiplett der aromatischen Protonen. Bei $\delta=6.72 \mathrm{ppm}$ ist ein breites, mit $\mathrm{D}_{2} \mathrm{O}$ austauschbares Signal erkennbar, welches dem NH-Proton zugeordnet wird. Infolge einer Kopplung ( $\mathrm{J}=5 \mathrm{~Hz}$ ) mit dem benachbarten NH-Proton spaltet das Signal der beiden Methylenprotonen in ein Dublett auf, welches bei $\delta=4.19$ ppm registriert wird. Bei Zugabe von $\mathrm{d}_{4^{-}}$ Methanol wird anstelle des Dubletts ein Singulett sichtbar. Die Ester-Methylgruppe wird bei $\delta=3.75$ ppm, die am Aromaten gebundene Methylgruppe bei $\delta=2.35 \mathrm{ppm}$ registriert. Im IR-Spektrum von 3Methylhippursäuremethylester ist bei $3340 \mathrm{~cm}^{-1}$ die $\mathrm{N}-\mathrm{H}$-Valenzschwingung erkennbạt. Die intensive 

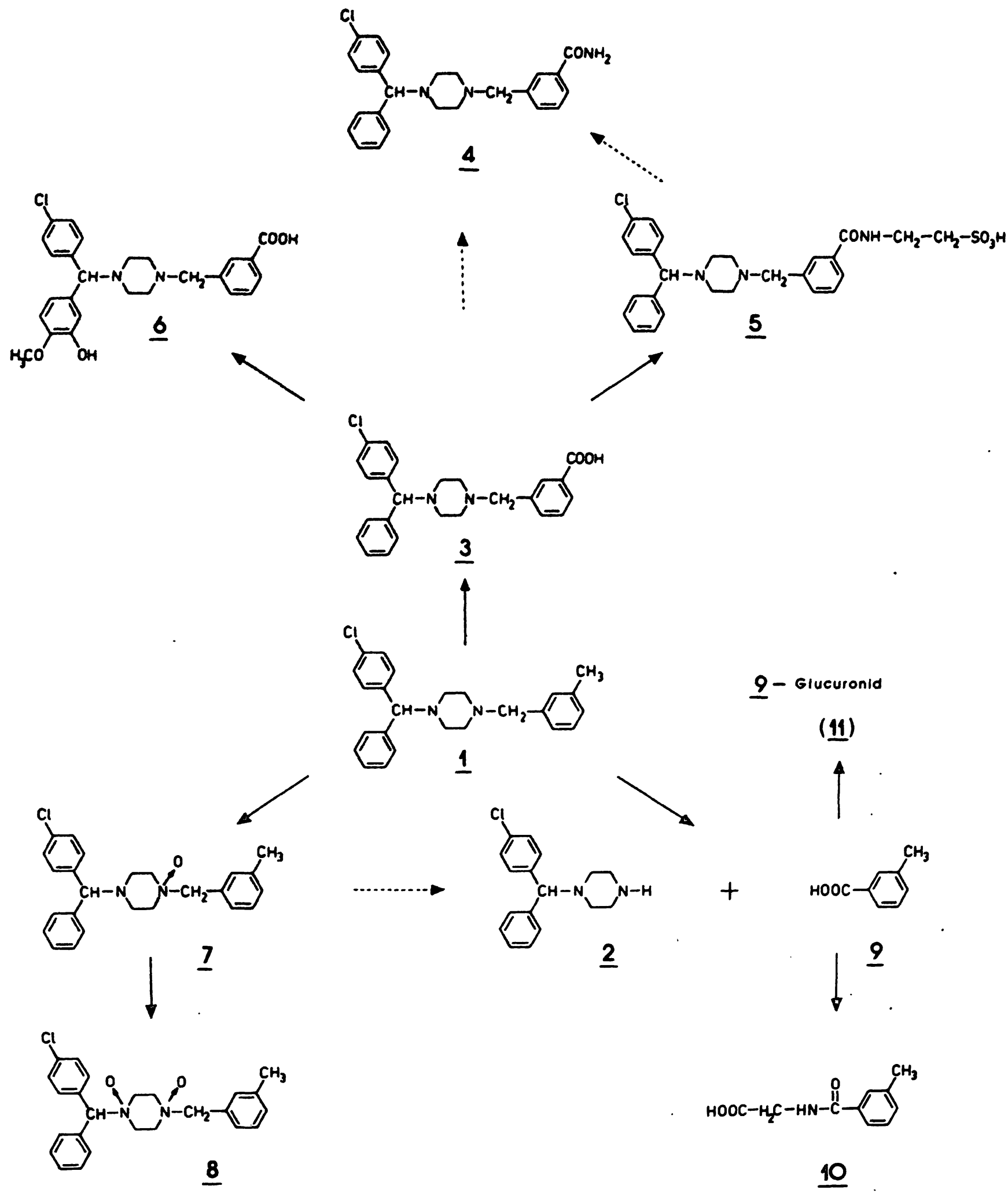

Abb. 9. Metabolisiẹrungsschema des Meclozins (1)

Bande bei $1760 \mathrm{~cm}^{-1}$ wird der $\mathrm{C}=\mathrm{O}$-Valenzschwingung des Esters zugeordnet. Bei $1660 \mathrm{~cm}^{-1}$ und 1540 $\mathrm{cm}^{-1}$ werden die $\mathrm{C}=\mathrm{O}$-Valenz- und NH-Deformationsschwingung des Sãureamids registriert. Das in Methanol aufgenommene UV-Spektrum des Methylesters von 10 zeigt Absorptionsmaxima bei $\lambda=230$ und $276 \mathrm{~nm}$.
Nach enzymatischer Glucuronidspaltung konnte im sauren Harnextrakt Metabolit 9 sowohl dünnschichtchromatographisch als auch gaschromatographisch nachgewiesen werden. Es wird daher angenommen, daß 9 zum Teil als 3-Methylbenzoesäure-Glucuronid (11) (Abb. 9) ausgeschieden wird. 
Im Harn und Fäces konnte ein weiteres hochpolares Abbauprodukt des Meclozins (I) dünnschichtchromatographisch detektiert werden. Im Massenspektrometer ließ sich diese Verbindung nicht unzersetzt verdampfen; im FAB-Spektrum konnte keine eindeutige Zuordnung des Molekülions erfolgen. Aufgrund der beobachteten Fragmentierungen wird angenommen, da $\beta$ es sich um ein Konjugat des Metaboliten 2 handelt. Hinweise auf eine weitere Metabolisierung von $\mathrm{N}$-[(4-Chlorphenyl)-phenylmethyl]-piperazin (2) sind auch in der Literatur zu finden (20). Aufgrund der geringen Menge war eine Identifizierung dieser Verbindung nicht möglich.

\section{Diskussion}

Nach Einnahme von Meclozin-dihydrochlorid (1diHCl) in Form von Postafen ${ }^{\circledR}$-Tabletten wird der weitaus größte Anteil des Arzneistoffes in unveränderter Form mit den Fäces ausgeschieden (Tab. 1). Aufgrund der identifizierten Metaboliten wird für Meclozin (1) das in Abbildung 9 angegebene Metabolisierungsschema angenommen. Demnach ist der Abbau des Meclozins (1) im menschlichen Körper gekennzeichnet durch:

- N-Oxid-Bildung

- Oxidative Desaminierung

- Oxidation der am Aromaten gebundenen Methylgruppe

- Aromatische Hydroxylierung

- O-Methylierung

- Konjugation mit Glucuronsäure

- Konjugation mit Aminosäuren

Die Bildung von N-Oxiden wurde bei vielen Arzneistoffen ähnlicher Struktur beobachtet (21). Jedoch konnte bisher kein substituiertes Piperazin-N,N'-dioxid im Rahmen der in der Literatur beschriebenen Metabolismus-Studien nachgewiesen werden.

Über den Mechanismus einer oxidativen Desaminierung kann die Bildung von $\mathrm{N}-[(4-$ Chlorphenyl)-phenylmethyl]-piperazin (2) erklärt werden. Die Bildung von 2 ist eine für viele strukturverwandte Verbindungen des Meclozins (l) beobachtete metabolische Reaktion (21); sie ist von großem Interesse, da für 2 an der Ratte eine teratogene Aktivität nachgewiesen wurde $(1,22)$.

Als zweites Reaktionsprodukt der oxidativen Desaminierung konnte 3-Methylbenzoesäure (9) nachgewiesen werden. Ein geringer Teil dieser Verbindung wird als Glucuronsäure-Konjugat (11) ausgeschieden; der weitaus größte Anteil wird jedoch nach Konjugation mit der Aminosäure Glycin als 3-Methylhippursäure (10) eliminiert.
Die metabolische Oxidation der am Aromaten gebundenen Methylgruppe führt zu 3-\{4-[(4-Chlorphenyl)phenylmethyl]-piperazino\}-methylbenzoesäure (3), einem zentralen Hauptabbauprodukt des Meclozins (1) (Abb. 9). Die Verbindung wird wahrscheinlich wegen der hohen Molekülmasse und der hohen Polarität hauptsächlich über die Fäcés ausgeschieden; im Harn konnten nur Spuren nachgewiesen werden (Tab. 1). Eine bei vielen aromatischen Carbonsäuren beobachtete Glycin-Konjugation konnte für 3 nicht nachgewiesen werden. Vielmehr wurde eine sehr ungewöhnliche Konjugation mit der Aminosäure Taurin beobachtet. Die wohl wichtigste biochemische Funktion dieser $\beta$-Aminosulfonsäure im menschlichen Organismus besteht in der Konjugation der Gallensäuren. Eine Bindung von Taurin an körperfremde Substanzen wurde bislang nur selten und fastusschließlich bei Tieren beobachtet (23). Eini seltenes Beispiel für ein aus menschlichem Harn isoliertes Taurin-Derivat stellt das von Grassi und Mitarbeitern identifizierte Taurin-Konjugat der Biphenylessigsäure dar (24). Die Bindung von Taurin erfolgt zumeist an aktivierte Arylessigsäuren (23); eine Konjugation mit Benzoesäure-Derivaten ist in der Literatur bislang nicht beschrieben. Es kann angenommen werden, daß eine derartige Taurin-Konjugation eine Alternative zu den im menschlichen Organismus üblichen Glycinund Glutamin-Konjugationen darstellt.

Die Bildung von 3-\{4-[(4-Chlorphenyl)-3'-hydroxy4'-methoxyphenylmethyl]-piperazino\}-methylbenzoesäure (6) spielt im Metabolismus von Meclozin (I) nur eine untergeordnete Rolle (Tab. 1). Als Vorstufe von 6 wird ein $o$-Diphenol angenommen, welches in einer Phase-II-Reaktion methyliert wird. Allerdings konnte weder das o-Diphenol noch das mono-hydroxylierte Produkt nachgewiesen werden.

Unklar ist die Entstehung des Säureamids 4. Daß es sich bei dieser Verbindung um ein bei der Aufarbeitung gebildetes Artefakt handelt, konnte durch Extraktionsversuche ausgeschlossen werden. Analog der Biogenese einiger Peptid-Hormone mit endständiger Säureamid-Gruppe (25) wird für 4 eine enzymatische Bildung aus einem Aminosäure-Konjugat vermutet.

Mindestens ein weiteres Abbauprodukt des Meclozins (I) konnte aus Harn und Fäces isoliert werden, welches jedoch nicht identifiziert werden konnte. Dabei handelt es sich möglicherweise um ein Konjugat der Verbindung 2.

Der durch Spaltung des Piperazin-Ringes èntstandene und aus Rattenorganen nach Meclozin (1)-Applikation isolierte Metabolit, $\mathrm{N}-[(4-\mathrm{Chlorphenyl)}$-phenylmethyll-ethylendiamin (2), konnte im menschlichen Harn und Stụhl nicht nachgewiesen iwerden. 


\section{Danksagung}

Wir danken der Deutschen Forschungsgemeinschaft für die Überlassung der Finnigan 212/188 MS-DS-Kombination.

Der Firma UCB Chemie GmbH möchten wir für die Überlassung von Meclozin-Vergleichssubstanz danken.

Dem Verband der Chemischen Industrie e. V., Fonds der Chemischen Industrie, danken wir für die geleistete Hilfe.

\section{Literatur}

1. Narrod, S. A., Wilk, A. L. \& King, C. T. G. (1965) J. Pharmacol. Exp. Therapeut. 147, 380-384.

2. Gaertner, H. J., Breyer, U. \& Liomin, G. (1973) J. Pharmacol. Exp. Therapeut. 185, 195-201.

3. Kato, K. Yoshida, K., Tatsumi, K. \& Tsukamoto, H. (1962) Chem durm. Bull. 10, 1238-1242.

4. Goe? Clin. Chcis!4Clin Brochem. 15, 489-498.

5. Merck, E. (1986, , nfärbereagenzien für Dünnschicht- und Papierchromatographie, Darmstadt.

6. Moll, F. (1974) Biochemische und Biopharmazeutische Untersuchungsverfahren, Wissenschaftliche Verlagsgesellschaft mbH, Stuttgart.

7. Wotschokowsky, M. (1980) Kombinations- und Mikrotechniken zur Strukturaufklärung im Mikrogrammbereich unter besonderer Berücksichtigung chromatographischer Trennmethoden, Merck, Darmstadt.

8. DeBoer, Th. J. \& Backer, H. J. (1956) Org. Synth. 36, 16-19.

9. Gleditsch, A. \& Moeller, H. (1889) Liebigs Ann. Chem. 250, 376-380.

10. Goenechea, S., Rücker, G., Brzezinka, H., Hoffmann, G., Neugebauer, M. \& Glanzmann, G., in Vorbereitung.

11. Glanzmann, G. (1987) Dissertation, Bonn.

12. Pfleger, K., Maurer, H. \& Weber, A. (1985) Mass Spectra and GC-Data of Drugs, Poisons and their Metabolites, $\mathrm{VCH}$ Verlagsgesellschaft $\mathrm{mbH}$, Weinheim.

13. Clarke, E. G. C. (1986) Isolation and Identification of Drugs, The Pharmaceutical Press, London.
Für die Aufnahme der FAB-Massenspcktren möchten wir Herrn Prof. Dr. H. Budzikiewicz und Herrn $H$. Münster, Institut für Organische Chemie der Universität Köln, unseren Dank aussprechen.

Wir danken Herrn $C$. Schmidt und Frau $R$. Leven, Zentralanalytik der Chemischen Institute der Universität Bonn, für die Aufnahme der 'H-NMR-Spektren.

14. Auterhoff, H. \& Kovar, K. A. (1985) Identifizierung von Arzneistoffen, 5. Aufl., Wissenschaftliche Verlagsgesellschaft mbH, Stuttgart.

15. Kamm, J. J., Szuna, A. \& Kuntzmann, R. (1972) J. Pharmacol. Exp. Therapeut. 182, 507-514.

16. Pouchert, C. J. (1975) The Aldrich Library of Infrared Spectra, 2. Aufl., Aldrich Chemical Co. Milwaukee USA.

17. Pouchert, C. J. \& Campbell, J. R. (1974) The Aldrich Library of NMR-Spectra, Aldrich Chemical Co. Milwaukee USA.

18. Ciaccio, L. L., Missan, S. R., McMullen, W. H. \& Grenfell, T. C. (1957) Anal. Chem. 29, 1670-1673.

19. Kira, S. (1977) Brit. J. Industr. Med. 34, 305-309.

20. Close, J. A., Gobert, J. G. \& Rodriguez, L. A. M. (1968) Proc. Eur. Soc. Study Drug Toxicity 9, 144-155.

21. Pfeiffer, S. \& Borchert, H.-H. (1977-1983) Biotransformation von Arzneimitteln, Verlag Chemie, Weinheim, Deerfield Beach-Florida, Basel.

22. King, C. T. G. (1963) Science $141,353-355$

23. The Royal Society of Chemistry (1970-1981) Foreign Compounds Metabolism in Mammals, Vol. 1-6, Burlington House, London.

24. Grassi, E., Passetti, G. L., Trebbi, A. \& Frigero, A. (1976) Mass Spectrom. Drug Metab. (Proc. Int. Symp.), pp. 95110.

25. Bradbury, A. F.; Finnie, M. D. A. \& Smyth, D. G. (1982) Nature 298, 686-688.

Prof. Dr. S. Goenechea Institut für Rechtsmedizin der Universität Bonn

Stiftsplatz 12

D-5300 Bonn 1 


\section{$\therefore 1$}

$$
y^{3}
$$

